$11-1-2011$

\title{
Synthesis and Antiproliferative Activity of Some Steroidal Lactams
}

Yanmin Huang

Guangxi Teachers Education University

Jianguo Cui

Guangxi Teachers Education University

Sijing Chen

Guangxi Teachers Education University

Chunfang Gan

Guangxi Teachers Education University

Aimin Zhou

Cleveland State University, A.ZHOU@csuohio.edu

Follow this and additional works at: https://engagedscholarship.csuohio.edu/scichem_facpub

Part of the Chemistry Commons

How does access to this work benefit you? Let us know!

\section{Recommended Citation}

Huang, Yanmin; Cui, Jianguo; Chen, Sijing; Gan, Chunfang; and Zhou, Aimin, "Synthesis and Antiproliferative Activity of Some Steroidal Lactams" (2011). Chemistry Faculty Publications. 412. https://engagedscholarship.csuohio.edu/scichem_facpub/412

This Article is brought to you for free and open access by the Chemistry Department at EngagedScholarship@CSU. It has been accepted for inclusion in Chemistry Faculty Publications by an authorized administrator of EngagedScholarship@CSU. For more information, please contact library.es@csuohio.edu. 


\title{
Synthesis and antiproliferative activity of some steroidal lactams
}

\author{
Yanmin Huang , Jianguo Cui , Sijing Chen , Chunfang Gan , Aimin Zhou
}

\section{Introduction}

Steroidal compounds display a variety of biological functions and play a very important role in life [1,2]. The steroidal drugs are widely used in traditional medicines, such as antibacterium, hormone kind medication, etc. The introduction of heteroatom or replacement of one or more carbon atoms in steroidal molecule by a heteroatom affects the chemical properties of the steroidal molecule and often results in alterations of its biological activities. The study of natural products which isolated from marine life showed that the steroidal compounds bearing different functional groups, such as hydroxyl, hydroximino, hydrazone, sulfate groups, had excellent cytotoxicity against some tumor cells [3 7].

A variety of steroids with unusual and interesting structures have been synthesized and evaluated for their anti tumor activity [8 10]. In order to evaluate the anti tumor activity of new steroi dal derivatives, we synthesized a series of steroidal oxime deriva tives and investigated their cytotoxic activity against different types of cancer cells $[11,12]$. Interestingly we found that the cyto toxic activity of a steroidal oxime is dependent on the cholesteric side chain and function groups at position 3 and 6 on the steroidal nucleus.

Azahomosteroids are also a class of steroid compounds which were synthesized and modified in order to increase biological activity of steroids. These compounds have been tested success fully as anti cancer drugs against several types of cancer cells [13 17]. In order to find novel and effective anti tumor agents, we synthesized a series of $17 \mathrm{a}$ aza $\mathrm{D}$ Homo andrester 17 one, 3 aza A homo 4 one bile acid and 7 deoxycholic acid derivatives with various groups on the steroidal nucleus, and the results showed that these compounds could exhibit a high cytotoxicity to HeLa tumor cell line in vitro $[18,19]$. Here, some steroidal com pounds carrying lactam at A ring and a different active group on the 6 position of steroidal nucleus were synthesized and evaluated for their antiproliferative activity against some cancer cells.

\section{Experimental}

Chemistry

The sterols and $\mathrm{NaBH}_{4}$ were purchased from the Merck Co. All chemicals and solvents were analytical grade and solvents were purified by general methods before being used. Melting points were determined on an $\mathrm{X}_{4}$ apparatus and were uncorrected. Infra red spectra were measured with a Nicolet FT 360 Spectrophotom eter. The ${ }^{1} \mathrm{H}$ and ${ }^{13} \mathrm{C}$ NMR spectra were recorded in $\mathrm{CDCl}_{3}$ on a Bruker AV 600 spectrometer at working frequencies 600 and $150 \mathrm{MHz}$ and a Bruker AV 300 spectrometer at working frequen cies 300 and $75 \mathrm{MHz}$, respectively. Chemical shifts are expressed in parts per million $(\delta)$ values and coupling constants $(J)$ in Hertz. LREIMS were recorded on a Thermo DSQ instrument. The cell pro liferation assay was undertaken by a MTT method using 96 well plates on Biocell ELISA analysis spectrometer. 
(3E) Hydroximinocholest 6 one (1) was prepared according to procedures in the literature [12].

The synthesis of 4 aza A homocholest 3,6 dione (2) and 3 aza A homocholest 3,6 dione (3)

The solution of thionyl chloride $(2.1 \mathrm{~mL})$ in $5 \mathrm{~mL}$ dry THF was added to a solution of oxime $1(450 \mathrm{mg}, 0.99 \mathrm{mmol})$ in dry THF $(15 \mathrm{~mL})$. The solution was stirred under anhydrous conditions for $1 \mathrm{~h}$ at $0{ }^{\circ} \mathrm{C}$. Then the reaction was terminated and water was added to the solution. The solution was neutralized with ammonia and the product was extracted with $\mathrm{CH}_{2} \mathrm{Cl}_{2}(20 \times 3 \mathrm{~mL})$. The combined extract was washed with water, $5 \% \mathrm{NaHCO}_{3}$, and saturated brine, dried over anhydrous $\mathrm{Na}_{2} \mathrm{SO}_{4}$ and evaporated under reduced pres sure to give a crude product ( $750 \mathrm{mg}$ ) which was chromatographed on silica gel (elution: petroleum ether $\left.\left(6090^{\circ} \mathrm{C}\right) / \operatorname{EtOAc}(1: 5)\right)$ to give a yellow oily mixture. The oily mixture was further subjected to chromatography (methanol/dichloromethane $(1: 20)$ ) to afford $184.8 \mathrm{mg}$ of 4 aza A homocholest 3,7 dione (2) as white crystals, yield: $45 \%, \theta_{\mathrm{mp}} 212213^{\circ} \mathrm{C}$; IR( $\left.\mathrm{KBr}\right) v / \mathrm{cm}^{-1}: 3190,3072,2950$, $2850,2310,1711,1687,1454,1380,816 ;{ }^{1} \mathrm{H} \quad \mathrm{NMR}\left(\mathrm{CDCl}_{3}\right.$, $600 \mathrm{MHz}) \delta: 0.671\left(3 \mathrm{H}, \mathrm{s}, 18 \mathrm{CH}_{3}\right), 0.862\left(3 \mathrm{H}, \mathrm{s}, 19 \mathrm{CH}_{3}\right), 0.864(3 \mathrm{H}$, $\mathrm{d}, J=6.6,26$ or $\left.27 \mathrm{CH}_{3}\right), 0.868\left(3 \mathrm{H}, \mathrm{d}, J=6.6,26\right.$ or $\left.27 \mathrm{CH}_{3}\right)$, $0.913\left(3 \mathrm{H}, \mathrm{d}, J=6.6,21 \mathrm{CH}_{3}\right), 1.998\left(1 \mathrm{H}, \mathrm{t}, J=12.6, \mathrm{C}_{1} \alpha \mathrm{H}\right)$, $2.054\left(1 \mathrm{H}, \mathrm{dt}, J=12.6,3.6, \mathrm{C}_{7} \beta \mathrm{H}\right), 2.305\left(1 \mathrm{H}, \mathrm{dd}, J=15.0,7.8, \mathrm{C}_{2}\right.$ $\beta \mathrm{H}), 2.332\left(1 \mathrm{H}\right.$, dd, $\left.J=13.2,4.8, C_{7} \alpha \mathrm{H}\right), 2.415\left(1 \mathrm{H}, \mathrm{d}, J=9.6, C_{5}\right.$ $\alpha \mathrm{H}), 2.599\left(1 \mathrm{H}, \mathrm{t}, J=13.8, \mathrm{C}_{2} \alpha \mathrm{H}\right), 3.311(1 \mathrm{H}$, ddd, $J=16.2,9.6,4.8$, $\left.\mathrm{C}_{4 \mathrm{a}} \beta \mathrm{H}\right), 3.445\left(1 \mathrm{H}\right.$, ddd, $\left.J=16.2,7.8,1.2, \mathrm{C}_{4 \mathrm{a}} \alpha \mathrm{H}\right), 6.007(1 \mathrm{H}$, brt, $J=4.8, \mathrm{NH}) ;{ }^{13} \mathrm{C} \mathrm{NMR}\left(\mathrm{CDCl}_{3}, 150 \mathrm{MHz}\right) \delta: 210.5(\mathrm{C} 6), 178.1(\mathrm{C} \mathrm{3})$, 61.5(C 5), 56.7(C 14), 56.1(C 17), 53.7(C 9), 46.8(C 7), 44.3(C 13), 42.8(C 10), 39.5(C 12), 38.0(C 8), 36.8(C 4a), 36.1(C 22), 35.7(C 20), 34.8(C 1), 31.2(C 2), 28.00(C 25), 27.99(C 16), 23.9(C 15), 23.8(C 24), 22.8(C 23), 22.7(C 27), 22.5(C 26), 21.5(C 11), 18.6(C 21), 12.9(C 19), 12.0(C 18); ESI MS $m / z: 416(\mathrm{M}+1)^{+}$.

In the reaction, 3 aza A homocholest 3,7 dione (3) (the isomer of 2 ) was obtained as a byproduct in $21.5 \%$ yield ( $88 \mathrm{mg}), \theta_{\mathrm{mp}} 235$ $237^{\circ} \mathrm{C}$; IR(KBr) $v / \mathrm{cm}^{-1}$ : 3190, 3072, 2953, 1707, 1679, 1466, 1368; ${ }^{1} \mathrm{H} \mathrm{NMR}\left(\mathrm{CDCl}_{3}, 600 \mathrm{MHz}\right) \delta: 0.666\left(3 \mathrm{H}, \mathrm{s}, 18 \mathrm{CH}_{3}\right), 0.833(3 \mathrm{H}, \mathrm{s}, 19$ $\left.\mathrm{CH}_{3}\right), 0.860\left(3 \mathrm{H}, \mathrm{d}, J=6.6,26\right.$ or $\left.27 \mathrm{CH}_{3}\right), 0.871(3 \mathrm{H}, \mathrm{d}, J=6.6,26$ or $\left.27 \mathrm{CH}_{3}\right), 0.909\left(3 \mathrm{H}, \mathrm{d}, J=6.6,21 \mathrm{CH}_{3}\right), 2.022(1 \mathrm{H}, \mathrm{dd}, J=26.4,13.2$, $\left.C_{7} \beta \mathrm{H}\right), 2.372\left(1 \mathrm{H}, \mathrm{dd}, J=12.6,4.2, C_{7} \alpha \mathrm{H}\right), 2.534(1 \mathrm{H}, \mathrm{dd}, J=13.8$, $\left.11.4, \mathrm{C}_{4 \mathrm{a}} \beta \mathrm{H}\right), 2.600\left(1 \mathrm{H}, \mathrm{d}, J=11.4, \mathrm{C}_{4 \mathrm{a}} \alpha \mathrm{H}\right), 2.720(1 \mathrm{H}, \mathrm{dd}, J=14.4$, 2.4, $\left.C_{5} \alpha \mathrm{H}\right), 3.048\left(1 \mathrm{H}\right.$, dddd, $\left.J=15.6,7.8,6.0,1.8, C_{2} \alpha \mathrm{H}\right)$, $3.391\left(1 \mathrm{H}\right.$, ddd, $\left.J=15.6,12.0,4.2, \mathrm{C}_{2} \beta \mathrm{H}\right), 6.040(1 \mathrm{H}$, brs, $\mathrm{NH}) ;{ }^{13} \mathrm{C}$ $\mathrm{NMR}\left(\mathrm{CDCl}_{3}, 150 \mathrm{MHz}\right) \quad \delta: \quad 209.5(\mathrm{C} 6), \quad 177.5(\mathrm{C} \mathrm{3}), \quad 56.7(\mathrm{C} 14)$, 56.1(C 17), 54.9(C 5), 54.1(C 9), 46.9(C 7), 44.3(C 13), 42.8(C 10), 41.5(C 12), 39.52(C 1), 39.48(C 24), 38.0(C 8), 37.6(C 2), 36.1(C 22), 35.7(C 20), 30.6(C 4a), 28.00(C 25), 27.99(C 16), 23.9(C 15), 23.8(C 23), 22.8(C 27), 22.6(C 26), 21.8(C 11), 18.6(C 21), 12.9(C 19), 12.0(C 18); ESI MS $m / z$ : 416(M+1) .

6 Hydroxy 4 aza A homocholest 3 one (4)

To the stirred solution of $2(100 \mathrm{mg}, 0.24 \mathrm{mmol})$ in $\mathrm{CH}_{3} \mathrm{OH}$ $(15 \mathrm{~mL})$ was added $\mathrm{NaBH}_{4}(30 \mathrm{mg}, 0.79 \mathrm{mmol})$ in one time at room temperature. After $30 \mathrm{~min}$, the reaction was stopped. The solution was neutralized with $1 \mathrm{M} \mathrm{HCl}$. After evaporation of the majority of $\mathrm{MeOH}$ under reduced pressure, the residue was extracted with ethyl acetate $(3 \times 15 \mathrm{~mL})$. The organic layer was washed with cold water and saturated brines. After drying over anhydrous sodium sulfate, the solvent was removed under reduced pressure and a crude product $(90 \mathrm{mg})$ was obtained. After crystallization from methanol, the compound 4 was obtained as a white solid $(81 \mathrm{mg}$, $81 \%), \theta_{\mathrm{mp}} 228230^{\circ} \mathrm{C}$; IR(KBr) $v / \mathrm{cm}^{-1}: 3411,2933,2864,1662$, 1466, 1372, 1262, 1094, 1041, 804; ${ }^{1} \mathrm{H} \mathrm{NMR}\left(\mathrm{CDCl}_{3}, 600 \mathrm{MHz}\right) \delta$ : $0.695\left(3 \mathrm{H}, \mathrm{s}, 18 \mathrm{CH}_{3}\right), 0.863\left(3 \mathrm{H}, \mathrm{d}, J=6.6,26\right.$ or $\left.27 \mathrm{CH}_{3}\right)$, $0.867\left(3 \mathrm{H}, \mathrm{d}, J=6.6,26\right.$ or $\left.27 \mathrm{CH}_{3}\right), 0.906\left(3 \mathrm{H}, \mathrm{d}, J=6.6,21 \mathrm{CH}_{3}\right)$, 1.144(3H, s, $\left.19 \mathrm{CH}_{3}\right), 2.004\left(1 \mathrm{H}, \mathrm{dt}, J=12.6,3.6, \mathrm{C}_{2} \alpha \mathrm{H}\right), 2.137(1 \mathrm{H}$, dd, $\left.J=15.0,1.8, C_{2} \beta \mathrm{H}\right), 3.0132 .963\left(1 \mathrm{H}, \mathrm{m}, \mathrm{C}_{4 \mathrm{a}} \beta \mathrm{H}\right), 3.038(1 \mathrm{H}$, dd, $\left.J=14.4,10.8, C_{4 a} \alpha \mathrm{H}\right), 3.420\left(1 \mathrm{H}\right.$, ddd, $J=15.6,12.0,4.2, \mathrm{C}_{6}$ $\beta \mathrm{H}), \quad 3.958(1 \mathrm{H}, \quad \mathrm{d}, J=1.8, \quad \mathrm{NH}), \quad 5.827(1 \mathrm{H}, \quad$ brs, $\mathrm{OH}) ;{ }^{13} \mathrm{C}$ $\mathrm{NMR}\left(\mathrm{CDCl}_{3}, 150 \mathrm{MHz}\right) \delta: 178.1$ (C 3), 73.6(C 6), 56.3 (C 14), 56.1(C 17), 54.0(C 9), 45.8(C 5), 43.5(C 13), 42.4(C 10), 39.9(C 12), 39.8(C 24), 39.5(C 8), 38.8(C 7), 38.1(C 4a), 37.7(C 22), 36.2(C 20), 35.8(C 1), 29.5(C 2), 28.2(C 25), 28.0(C 16), 24.2(C 15), 23.9(C 23), 22.8(C 27), 22.6(C 26), 21.2(C 11), 18.7(C 21), 15.3(C 19), 12.1(C 18); ESI MS $m / z: 418(\mathrm{M}+1)^{+}$.

\section{Hydroxy 3 aza A homocholest 3 one $(\mathbf{8})$}

Compound $\mathbf{8}$ was prepared similarly according to the procedure of $\mathbf{4}$, but the compound $\mathbf{3}$ used as starting material instead of the compound 2. Yield: $90 \%, \theta_{\mathrm{mp}} 245247^{\circ} \mathrm{C}$; IR(KBr) $v / \mathrm{cm}^{-1}$ : 3378 , 2937, 2868, 1650, 1462, 1380, 1209, 1143, 1094, 1021; ${ }^{1} \mathrm{H}$ $\operatorname{NMR}\left(\mathrm{CDCl}_{3}, 600 \mathrm{MHz}\right) \delta: 0.697\left(3 \mathrm{H}, \mathrm{s}, 18 \mathrm{CH}_{3}\right), 0.863(3 \mathrm{H}, \mathrm{d}$, $J=6.6,26$ or $\left.27 \mathrm{CH}_{3}\right), 0.867\left(3 \mathrm{H}, \mathrm{d}, J=6.6,26\right.$ or $\left.27 \mathrm{CH}_{3}\right)$, $0.909\left(3 \mathrm{H}, \mathrm{d}, J=6.6,21 \mathrm{CH}_{3}\right), 1.152\left(3 \mathrm{H}, \mathrm{s}, 19 \mathrm{CH}_{3}\right), 2.270(1 \mathrm{H}, \mathrm{dd}$, $\left.J=15.0,8.4, \mathrm{C}_{4 \mathrm{a}} \alpha \mathrm{H}\right), 2.634\left(1 \mathrm{H}, \mathrm{t}, J=13.2, \mathrm{C}_{2} \alpha \mathrm{H}\right), 2.865(1 \mathrm{H}, \mathrm{dd}$, $\left.J=15.6,7.8, C_{2} \beta \mathrm{H}\right), 3.725\left(1 \mathrm{H}\right.$, ddd, $\left.J=15.6,9.3,4.2, C_{6} \beta \mathrm{H}\right)$, $3.984(1 \mathrm{H}, \mathrm{d}, J=1.8 \mathrm{~Hz}, \mathrm{NH}), 6.003(1 \mathrm{H}, \mathrm{brs}, \mathrm{OH}) ;{ }^{13} \mathrm{C} \mathrm{NMR}\left(\mathrm{CDCl}_{3}\right.$, $150 \mathrm{MHz}$ ) $\delta:$ 178.1(C 3), 73.6(C 6), 56.3 (C 14), 56.1(C 17), 54.0(C 9), 45.8(C 5), 43.5(C 13), 42.4(C 10), 39.9(C 12), 39.8(C 24), 39.5(C 8), 38.8(C 7), 38.1(C 4a), 37.7(C 22), 36.2(C 20), 35.8(C 1), 29.5(C 2), 28.2(C 25), 28.0(C 16), 24.2(C 15), 23.9(C 23), 22.8(C 27), 22.6(C 26), 21.2(C 11), 18.7(C 21), 15.3(C 19), 12.1(C 18); ESI MS $m / z$ : $418(\mathrm{M}+1)^{+}$.

6 Hydroximino 4 aza A homocholest 3 one (5)

Compound $2(100 \mathrm{mg}, 0.24 \mathrm{mmol})$ was dissolved in $10 \mathrm{~mL}$ of $95 \% \mathrm{CH}_{3} \mathrm{CH}_{2} \mathrm{OH}$. After the mixture was heated to $60^{\circ} \mathrm{C}, \mathrm{CH}_{3} \mathrm{COO}$ $\mathrm{Na} \cdot 3 \mathrm{H}_{2} \mathrm{O}(33 \mathrm{mg}, 0.24 \mathrm{mmol})$ and $\mathrm{NH}_{2} \mathrm{OH} \cdot \mathrm{HCl}(20 \mathrm{mg}, 0.27 \mathrm{mmol})$ were added into the solution in $10 \mathrm{~min}$. The mixture was stirred for $1 \mathrm{~h}$ at $60^{\circ} \mathrm{C}$. Then reaction was terminated and the majority of solvent was evaporated under reduced pressure. Distilled water was added into the reaction mixture, and the product was ex tracted with ethyl acetate. The combined extract was washed with saturated brine, dried with anhydrous sodium sulfate and evapo rated under reduce pressure. The residue was subject to chroma tography (methanol/dichloromethane $(1: 20)$ ) to produce $81 \mathrm{mg}$ of 5 (90\%), $\theta_{\mathrm{mp}} 243245^{\circ} \mathrm{C}$; IR( $\left.\mathrm{KBr}\right) v / \mathrm{cm}^{-1}: 3321,3240,2940$, 2864, 1654, 1470, 1380, 968; ${ }^{1} \mathrm{H} \operatorname{NMR}\left(\mathrm{CDCl}_{3}, 600 \mathrm{MHz}\right) \delta$ : $0.650\left(3 \mathrm{H}, \mathrm{s}, 18 \mathrm{CH}_{3}\right), 0.842\left(3 \mathrm{H}, \mathrm{s}, 19 \mathrm{CH}_{3}\right), 0.862(3 \mathrm{H}, \mathrm{d}, J=6.6$, 26 or $\left.27 \mathrm{CH}_{3}\right), 0.867\left(3 \mathrm{H}, \mathrm{d}, J=6.6,26\right.$ or $\left.27 \mathrm{CH}_{3}\right), 0.898(3 \mathrm{H}, \mathrm{d}$, $\left.J=6.6,21 \mathrm{CH}_{3}\right), 1.865\left(1 \mathrm{H}, \mathrm{dd}, J=15.0,6.0, \mathrm{C}_{5} \alpha \mathrm{H}\right), 2.001(1 \mathrm{H}, \mathrm{dt}$, $\left.J=12.0,3.0, C_{2} \alpha \mathrm{H}\right), 2.333\left(1 \mathrm{H}, \mathrm{d}, J=10.8, \mathrm{C}_{8} \alpha \mathrm{H}\right), 2.611(1 \mathrm{H}, \mathrm{dd}$, $\left.J=15.0,11.4, C_{2} \beta \mathrm{H}\right), 3.046\left(1 \mathrm{H}, \mathrm{d}, J=13.8, \mathrm{C}_{7} \alpha \mathrm{H}\right), 3.025(1 \mathrm{H}, \mathrm{dd}$, $\left.J=13.8,6.0, C_{7} \beta \mathrm{H}\right), 3.377\left(1 \mathrm{H}, \mathrm{dd}, J=13.2,4.8, \mathrm{C}_{4 \mathrm{a}} \alpha \mathrm{H}\right), 3.419(1 \mathrm{H}$, ddd, $\left.J=15.0,11.4,4.2, C_{4 a} \beta \mathrm{H}\right), 6.193(1 \mathrm{H}$, brs, $N H), 9.094(1 \mathrm{H}$, brs, $\mathrm{NOH}) ;{ }^{13} \mathrm{C} \mathrm{NMR}\left(\mathrm{CDCl}_{3}, 150 \mathrm{MHz}\right) \delta: 179.1(\mathrm{C} \mathrm{3}), 158.7(\mathrm{C} 6)$, 56.6(C 14), 56.2(C 17), 54.4(C 9), 54.0(C 5), 47.8(C 13), 42.8(C 10), 42.4(C 24), 41.3(C 4a), 39.8(C 12), 39.5(C 7), 37.8(C 8), 36.1(C 22), 35.7(C 20), 31.9(C 1), 29.9(C 2), 28.1(C 25), 28.0(C 16), 24.0(C 15), 23.8(C 23), 22.8(C 27), 22.6(C 26), 21.7(C 11), 18.6(C 21), 12.5(C 19), 12.0(C 18); ESI MS m/z: 431(M+1)+.

. 6 Hydroximino 3 aza A homocholest 3 one (9)

Compound $\mathbf{9}$ was prepared similarly according to the procedure of 5 using the compound 3 as the starting material. Yield: $81 \%, \theta_{\mathrm{mp}}$ $245257^{\circ} \mathrm{C}$; IR(KBr) $v / \mathrm{cm}^{-1}$ : 3119, 2929, 2859, 1658, 1470, 1381, 1135, 959, 910; ${ }^{1} \mathrm{H} \mathrm{NMR}\left(\mathrm{CDCl}_{3}, 600 \mathrm{MHz}\right) \delta: 0.589(3 \mathrm{H}, \mathrm{s}, 18$ $\left.\mathrm{CH}_{3}\right), 0.799\left(3 \mathrm{H}, \mathrm{s}, 19 \mathrm{CH}_{3}\right), 0.791\left(3 \mathrm{H}, \mathrm{d}, J=6.6 \mathrm{~Hz}, 26\right.$ or $\left.27 \mathrm{CH}_{3}\right)$, $0.807\left(3 \mathrm{H}, \mathrm{d}, J=6.6 \mathrm{~Hz}, 26\right.$ or $\left.27 \mathrm{CH}_{3}\right), 0.834(3 \mathrm{H}, \mathrm{d}, J=6.6 \mathrm{~Hz}, 21$ $\left.\mathrm{CH}_{3}\right), 1.880\left(1 \mathrm{H}, \mathrm{dd}, J=13.5,6.6 \mathrm{~Hz}, \mathrm{C}_{5} \alpha \mathrm{H}\right), 1.956\left(1 \mathrm{H}, \mathrm{m}, \mathrm{C}_{2} \alpha \mathrm{H}\right)$, $2.065\left(1 \mathrm{H}\right.$, brt $\left.J=9.0 \mathrm{~Hz}, \mathrm{C}_{8} \alpha \mathrm{H}\right), 2.167\left(1 \mathrm{H}, \mathrm{td}, J=14.4,7.8 \mathrm{~Hz}, \mathrm{C}_{8}\right.$ $\alpha \mathrm{H}), 2.572\left(1 \mathrm{H}, \mathrm{dd}, J=22.8,13.8 \mathrm{~Hz}, \mathrm{C}_{2} \beta \mathrm{H}\right), 3.238(1 \mathrm{H}, \mathrm{dd}, J=15.6$, 
9.6 Hz, $\left.\mathrm{C}_{4 \mathrm{a}} \alpha \mathrm{H}\right), 3.296\left(1 \mathrm{H}, \mathrm{m}, \mathrm{C}_{7} \beta \mathrm{H}\right), 3.423(1 \mathrm{H}, \mathrm{dd}, J=15.6,9.0 \mathrm{~Hz}$, $\left.\mathrm{C}_{4 \mathrm{a}} \beta \mathrm{H}\right), \quad 6.428(1 \mathrm{H}, \quad$ brs, $\mathrm{NH}), \quad 9.004(1 \mathrm{H}$, brs, $\mathrm{NOH}) ;{ }^{13} \mathrm{C}$ $\mathrm{NMR}\left(\mathrm{CDCl}_{3}, 150 \mathrm{MHz}\right) \delta: \quad 179.6(\mathrm{C} 4), 158.9(\mathrm{C} \mathrm{6}), \quad 56.5(\mathrm{C} 14)$, 56.0(C 17), 53.9(C 9), 53.7(C 5), 42.6(C 13), 42.1(C 24), 39.6(C 10), 39.4(C 12), 37.9(C 1), 36.0(C 22), 35.7(C 20), 35.6(C 2), 34.4(C 8), 30.7(C 4a), 29.5(C 7), 28.0(C 25), 27.9(C 16), 23.9(C 15), 23.7(C 23), 22.6(C 27), 22.4(C 26), 21.3(C 11), 18.4(C 21), 12.2(C 19), 11.9(C 18); ESI MS $m / z: 431(\mathrm{M}+1)^{+}$.

\section{4 aza A Homocholest 3,6 dione 6 thiosemicarbazone (6)}

A mixture of 4 aza A homocholest 3,6 dione (100 mg, $0.249 \mathrm{mmol}$ ), thiosemicarbazide $(124 \mathrm{mg}, 0.5 \mathrm{mmol}$ ), and a few drops of glacial acetic acid $(0.5 \mathrm{~mL})$ in $95 \%$ ethanol $(20 \mathrm{~mL})$ was stirred at $6070^{\circ} \mathrm{C}$ for $2 \mathrm{~h}$. After completion of the reaction, the majority of solvent was evaporated and some water was added to this solution. The mixture was extracted with $\mathrm{CH}_{2} \mathrm{Cl}_{2}$ and the ex tract was washed with saturated brine, dried with anhydrous so dium sulfate and evaporated under reduce pressure. The resulting residue was chromatographed on a column of silica gel with a mixture of DCM methanol $(20: 1)$ to give compound 6 (91 mg, 72\%), $\theta_{\mathrm{mp}} 228230^{\circ} \mathrm{C}$; IR( $\left.\mathrm{KBr}\right) v / \mathrm{cm}^{-1}: 3452,3338,2941$, $2859,1658,1580,1462,1350,1221,1150,1071,956 ;{ }^{1} \mathrm{H}$ $\mathrm{NMR}\left(\mathrm{CDCl}_{3}, 600 \mathrm{MHz}\right) \delta: 0.665\left(3 \mathrm{H}, \mathrm{s}, 18 \mathrm{CH}_{3}\right), 0.823(3 \mathrm{H}, \mathrm{s}, 19$ $\left.\mathrm{CH}_{3}\right), 0.867\left(3 \mathrm{H}, \mathrm{d}, J=6.6,26\right.$ or $\left.27 \mathrm{CH}_{3}\right), 0.872(3 \mathrm{H}, \mathrm{d}, J=6.6,26$ or $\left.27 \mathrm{CH}_{3}\right), 0.907\left(3 \mathrm{H}, \mathrm{d}, J=6.6,21 \mathrm{CH}_{3}\right), 1.975(1 \mathrm{H}$, dd, $J=14.4$, 8.4, $\left.C_{5} \alpha \mathrm{H}\right), 2.244\left(1 \mathrm{H}, \mathrm{d}, J=9.0, C_{7} \beta \mathrm{H}\right), 2.301(1 \mathrm{H}, \mathrm{ddd}, J=14.4$, 6.6, 1.8, $\left.C_{2} \beta \mathrm{H}\right), 2.597\left(1 \mathrm{H}, \mathrm{t}, J=13.8, \mathrm{C}_{1} \beta \mathrm{H}\right), 2.672(1 \mathrm{H}, \mathrm{dd}$, $\left.J=12.6,2.4, C_{2} \alpha \mathrm{H}\right), 3.396\left(1 \mathrm{H}, \mathrm{ddd}, J=15.0,9.6,4.8, C_{4 a} \beta \mathrm{H}\right)$, $3.490\left(1 \mathrm{H}, \mathrm{dd}, J=15.0,8.4, \mathrm{C}_{4 \mathrm{a}} \alpha \mathrm{H}\right), 6.082(1 \mathrm{H}$, brs, $(\mathrm{C}=\mathrm{S}) \mathrm{NH})$, 6.355 $(1 \mathrm{H}$, brs, $(\mathrm{C}=\mathrm{S}) \mathrm{NH}), 7.058(1 \mathrm{H}, \mathrm{d}, J=4.2, \quad(\mathrm{C}=\mathrm{O}) \mathrm{NH})$, $8.756(1 \mathrm{H}$, brs, $\quad \mathrm{N} \mathrm{NH} \mathrm{C}) ;{ }^{13} \mathrm{C} \mathrm{NMR}\left(\mathrm{CDCl}_{3}, 150 \mathrm{MHz}\right) \delta: 179.1($ $\mathrm{C}=\mathrm{S}$ ), 178.4(C 3), 155.5(C 6), 56.4(C 14), 56.1(C 17), 55.5(C 9), 54.1(C 5), 49.4(C 13), 43.2(C 4a), 42.9(C 24), 39.5(C 12), 38.5(C 8), 36.8(C 10), 36.1(C 22), 35.7(C 20), 34.8(C 1), 31.9(C 2), 30.9(C 7), 28.07(C 16), 28.02(C 25), 24.2(C 19), 23.8(C 15), 22.8(C 26), 22.6(C 27), 21.5(C 23), 18.6(C 11), 12.6(C 21), 12.0(C 18); ESI MS $m / z: 489$.

\section{4 aza A Homocholest 3,6 dione 6 semicarbazone (7)}

Compound 7 was prepared similarly according to the procedure of $\mathbf{6}$, but the semicarbazide used as an attack reagent instead of the thiosemicarbazide and the reaction mixture was heated at 70 $80{ }^{\circ} \mathrm{C}$ for $6 \mathrm{~h}$. Yield: $62 \% . \theta_{\mathrm{mp}} 219220^{\circ} \mathrm{C}$; $\mathrm{IR}(\mathrm{KBr}) v / \mathrm{cm}^{-1}: 3448$, 2949, 2868, 1654, 1576, 1470, 1376, $1227 ;{ }^{1} \mathrm{H} \operatorname{NMR}\left(\mathrm{CDCl}_{3}\right.$, $600 \mathrm{MHz}) \delta: 0.652\left(3 \mathrm{H}, \mathrm{s}, 18 \mathrm{CH}_{3}\right), 0.806\left(3 \mathrm{H}, \mathrm{s}, 19 \mathrm{CH}_{3}\right), 0.866(3 \mathrm{H}$, d, $J=6.6,26$ or $\left.27 \mathrm{CH}_{3}\right), 0.871\left(3 \mathrm{H}, \mathrm{d}, J=6.6,26\right.$ or $\left.27 \mathrm{CH}_{3}\right)$, $0.902\left(3 \mathrm{H}, \mathrm{d}, J=6.6,21 \mathrm{CH}_{3}\right), 1.880\left(1 \mathrm{H}, \mathrm{dd}, J=14.4,6.0, \mathrm{C}_{7} \alpha \mathrm{H}\right)$, $2.021\left(1 \mathrm{H}\right.$, ddd, $\left.J=12.6,6.0,3.6, \mathrm{C}_{1} \alpha \mathrm{H}\right), 2.389\left(1 \mathrm{H}, \mathrm{d}, J=10.8, \mathrm{C}_{5}\right.$ $\alpha \mathrm{H}), 2.656\left(1 \mathrm{H}, \mathrm{dd}, J=15.0,3.6, \mathrm{C}_{2} \beta \mathrm{H}\right), 2.827\left(1 \mathrm{H}, \mathrm{brd}, J=16.2, \mathrm{C}_{2}\right.$ $\alpha \mathrm{H}), 3.050\left(1 \mathrm{H}, \mathrm{m}, \mathrm{C}_{4 \mathrm{a}} \beta \mathrm{H}\right), 3.387\left(1 \mathrm{H}, \mathrm{dd}, J=12.0,4.2, \mathrm{C}_{4 \mathrm{a}} \alpha \mathrm{H}\right)$, $4.908(1 \mathrm{H}, \quad$ brs, $\quad \mathrm{NC}(\mathrm{O}) \mathrm{NH}), \quad 6.021(1 \mathrm{H}, \quad$ brs, $\mathrm{NC}(\mathrm{O}) \mathrm{NH})$, $6.264(1 \mathrm{H}, \mathrm{d}, J=4.2, \quad(\mathrm{C}=\mathrm{O}) \mathrm{NH}), 7.995(1 \mathrm{H}, \mathrm{s}, \quad \mathrm{N} \mathrm{NH} \mathrm{C}) ;{ }^{13} \mathrm{C}$ $\mathrm{NMR}\left(\mathrm{CDCl}_{3}, \quad 150 \mathrm{MHz}\right) \quad \delta: \quad 178.0(\mathrm{C} \mathrm{3}), \quad 157.8(\mathrm{C} 6), \quad 152.0($ $\mathrm{NC}(\mathrm{O}) \mathrm{NH}_{2}$ ), 56.3(C 14), 56.1(C 17), 54.4(C 9), 49.2(C 5), 42.9(C 13), 42.8(C 4a), 41.4(C 10), 39.6(C 12), 39.5(C 24), 37.6(C 8), 36.2(C 2), 36.1(C 22), 35.7(C 20), 32.6(C 1), 31.7(C 7), 28.1(C 16), 28.0(C 25), 24.1(C 19), 23.8(C 15), 22.8(C 26), 22.6(C 27), 21.7(C 23), 18.6(C 11), 12.5(C 21), 12.0(C 18); ESI MS $m / z$ : 473(M+1) ${ }^{+}$.

\section{Antiproliferative activity}

Material and methods

Stock solutions of the compounds were prepared in sterile di methyl sulfoxide (DMSO) (Sigma) at a concentration of $10 \mathrm{mg} / \mathrm{mL}$ and afterward diluted with complete nutrient medium (RPMI
1640) supplemented with $10 \%$ heat inactivated fetal bovine serum and $0.1 \mathrm{~g} / \mathrm{L}$ penicillin $\mathrm{G}+0.1 \mathrm{~g} / \mathrm{L}$ streptomycin sulfate.

\section{Cell culture}

SMMC 7404 (human liver carcinoma), MGC 7901 (human gas tric carcinoma), HeLa (human cervical carcinoma) cancer cells were grown in the medium (RPMI 1640) supplemented with $10 \%$ cosmic calf serum and $0.1 \mathrm{~g} / \mathrm{L}$ penicillin $\mathrm{G}+0.1 \mathrm{~g} / \mathrm{L}$ streptomycin sulfate in a humidified atmosphere of $5 \% \mathrm{CO}_{2}$ at $37{ }^{\circ} \mathrm{C}$.

\section{Assay for cell viability}

The viability of these cells was determined using MTT (3 (4,5 dimethylthiazol 2 yl) 2,5 diphenyltetrazolium bromide) dye reduction assay. Briefly, cells $\left(1 \times 10^{4}\right.$ cells per well $)$ were seeded in 96 well plates. One day after seeding, the cells were treated with different concentration of each compound. An equal amount of DMSO was added to the cells used as negative controls. All were treated in triplicate. After reincubated for $72 \mathrm{~h}$, the cells were washed with sterile phosphate buffer saline (PBS). The $190 \mu \mathrm{L}$ of RPMI 1640 and $10 \mu \mathrm{L}$ of the tetrazolium dye (MTT) $(5 \mathrm{mg} / \mathrm{mL})$ solution were added to each well, and the cells were incubated for an additional $4 \mathrm{~h}$. The medium was discarded and $200 \mu \mathrm{L}$ of DMSO was added to dissolve the purple formazan crystals formed. The absorbance $(A)$ at $492 \mathrm{~nm}$ was measured using a Biocell ELISA analysis spectrometer. The $\mathrm{IC}_{50}$ value was calculated as the concen tration of drug yielding 50\% cell survival. The effect of compounds on the morphology of treated human carcinoma cells was investi gated by the light microscope and then photographed by $\mathrm{Ni}$ kon(TE2000 U) inverted microscope.

\section{Results and discussion}

\section{Chemistry}

Scheme 1 outlines the synthetic procedures of compounds $2 \mathbf{9}$. In our previous report [12], compound $\mathbf{1}$ was obtained in $66.5 \%$ yield by four steps using cholesterol as a starting material. The structure of compound $\mathbf{1}$ had been confirmed by its IR and NMR spectra.

The compounds 2 and $\mathbf{3}$ were synthesized by Beckmann rear rangement of 1 with $\mathrm{SOCl}_{2} / \mathrm{THF}$ at $0{ }^{\circ} \mathrm{C}$. In the reaction, the com pound 2 with 4 aza structure was obtained as a major product in $45 \%$ yield. At the same time, the compound 3 with 3 aza structure was obtained as a byproduct in 21.5\% yield. The structures of $\mathbf{2}$ and $\mathbf{3}$ were confirmed by analysis of the proton and carbon NMR chem ical shifts at $2 \mathrm{C}$ and $4 \mathrm{a} \mathrm{C}$. Resonances showing of $\mathrm{C}_{4 \mathrm{a}} \beta \mathrm{H}$ at $3.311 \mathrm{ppm}$ (ddd, $J=16.2,9.6$ and $4.8 \mathrm{~Hz}$ ) and $\mathrm{C}_{4 \mathrm{a}} \alpha \mathrm{H}$ at $3.445 \mathrm{ppm}$ (ddd, $J=16.2,7.8,1.2 \mathrm{~Hz}$ ) demonstrated a position of $4 \mathrm{NH}$ in the compound 2 , while the chemical shifts found for $\mathrm{C}_{2}$ $\alpha \mathrm{H}$ at $3.048 \mathrm{ppm}$ (dddd, $J=15.6,7.8,6.0,1.8 \mathrm{~Hz}$ ) and $\mathrm{C}_{2} \beta \mathrm{H}$ at $3.391 \mathrm{ppm}$ (ddd, $J=15.6,12.0,4.2 \mathrm{~Hz}$ ) were an indicative of the $3 \mathrm{NH}$ in the compound 3. Here, the amide protons produced a cou pling effect to $\mathrm{C}_{4} \mathrm{H}$ in 2 and $\mathrm{C}_{2} \mathrm{H}$ in 3, respectively. Moreover, ${ }^{1} \mathrm{H}$ NMR revealed the presence of the broad singlet at $6.007 \mathrm{ppm}$ in $\mathbf{2}$ and $6.040 \mathrm{ppm}$ in $\mathbf{3}$ for the amide proton.

Compounds 4 and 8 were obtained by reduction using $\mathrm{NaBH}_{4}$ as reductant in $\mathrm{CH}_{3} \mathrm{OH}$. The structures of compounds $\mathbf{4}$ and $\mathbf{8}$ were de duced from its analytical and spectral data. In the ${ }^{1} \mathrm{H}$ NMR spec trum, the resonances showing of $\mathrm{C}_{6} \beta \mathrm{H}$ at $3.420 \mathrm{ppm}$ (ddd, $J=15.6,12.0,4.2 \mathrm{~Hz}$ ) and $6 \mathrm{C}$ at $73.6 \mathrm{ppm}$ for 4 and $C_{6} \beta \mathrm{H}$ at $3.725 \mathrm{ppm}$ (ddd, $J=15.6,9.3,4.2 \mathrm{~Hz}$ ) and $6 \mathrm{C}$ at $73.6 \mathrm{ppm}$ for $\mathbf{8}$ demonstrated a position of 6 hydroxy respectively. In IR spectrum, the absorption peaks at $34113378 \mathrm{~cm}^{-1}$ showed that 6 carbonyl had been converted to 6 hydroxy in $\mathbf{4}$ and 8 . 


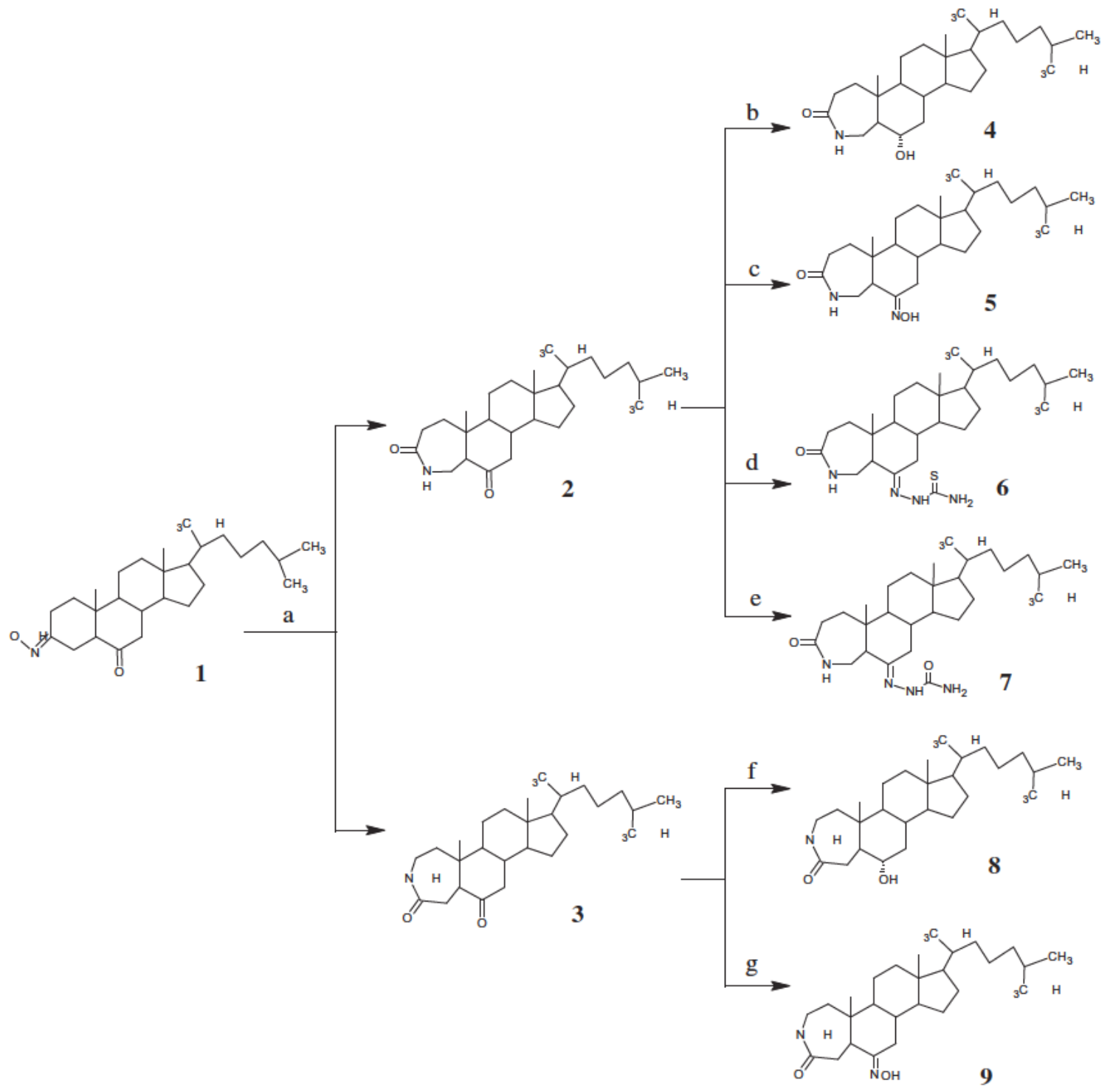

Scheme 1. Reagents and conditions: (a) $\mathrm{SOCl}_{2} / \mathrm{THF}, 0^{\circ} \mathrm{C}$; (b) $\mathrm{NaBH}_{4} / \mathrm{MeOH}$, rt; (c) $\mathrm{H}_{2} \mathrm{NOH} \cdot \mathrm{HCl} / \mathrm{Na}_{2} \mathrm{AC}_{3} \cdot 3 \mathrm{H}_{2} \mathrm{O} / \mathrm{EtOH}$, reflux; (d) $\mathrm{H}_{2} \mathrm{NC}(\mathrm{S}) \mathrm{NHNH} / \mathrm{EtOH}, 60{ }^{\circ} \mathrm{C}$; (e) $\mathrm{H}_{2} \mathrm{NC}(\mathrm{O}) \mathrm{NHNH}_{2} /$ EtOH; (f) $\mathrm{NaBH}_{4} / \mathrm{MeOH}$, rt; (g) $\mathrm{H}_{2} \mathrm{NOH} \cdot \mathrm{HCl} / \mathrm{Na}_{2} \mathrm{Ac} \cdot 3 \mathrm{H}_{2} \mathrm{O} / \mathrm{EtOH}$, reflux.

Compounds 5 and 9 were synthesized by the oximation of 2 and 3. The structures of $\mathbf{5}$ and $\mathbf{9}$ were confirmed by analysis of IR, ${ }^{1} \mathrm{H}$ and ${ }^{13} \mathrm{C}$ NMR chemical shifts at $7 \mathrm{C}$. In the IR spectra, the absorp tions of 1711 and $1707 \mathrm{~cm}^{-1}$ for the original carbonyl group in 2 and 3 , were absent and replaced by a new absorption at $1654 \mathrm{~cm}^{-1}(\mathrm{C}=\mathrm{N})$ for 5 and $1658 \mathrm{~cm}^{-1}$ for 9 . IR spectra bands at $3321 \mathrm{~cm}^{-1}$ in 5 and $3119 \mathrm{~cm}^{-1}$ in 9 indicate the presence of a hydroximino group. In the ${ }^{1} \mathrm{H}$ NMR spectrum of compounds 5 and 9, the signal for $\mathrm{C}_{7} \beta \mathrm{H}$ was shifted downfield, appearing at $3.025 \mathrm{ppm}$ for 5 and $3.296 \mathrm{ppm}$ for $\mathbf{9}$ due to the deshielding influ ence of the hydroxyl oxygen of the oxime which confirmed the $(E)$ configuration [20]. Also, the ${ }^{1} \mathrm{H}$ NMR revealed the presence of broad singlet $(1 \mathrm{H})$ at $9.094 \mathrm{ppm}$ in $\mathbf{5}$ and at $9.004 \mathrm{ppm}$ in $\mathbf{9}$ for the $\mathrm{NOH}$ group.

Similarly, the reaction of compound $\mathbf{2}$ with thiosemicarbazide or semicarbazide using few drops of glacial acetic acid as a catalyst afforded the corresponding product 6 or 7 . In the IR spectra, the compound 6 showed intense bands in the region $1150 \mathrm{~cm}^{-1}$ due to the $v(\mathrm{C}=\mathrm{S})$ stretching of the thiocarboxamide group. In addition, the absorption bands at $1580 \mathrm{~cm}^{-1}$ were attributed to the $v(C=N)$ stretching vibration, which also confirms the formation of desired thiosemicarbazone in the compound $\mathbf{6}$. Similarly, the compound 7 showed an absorption band of the $v(\mathrm{C}=\mathrm{N})$ stretching vibration at $1576 \mathrm{~cm}^{-1}$. The compounds 6 and 7 showed additional sharp bands in the region $34523338 \mathrm{~cm}^{-1}$ due to the $v(\mathrm{~N} \mathrm{H})$ stretching vibration. In the ${ }^{1} \mathrm{H}$ NMR spectrum, the singlets appearing at $8.756 \mathrm{ppm}$ for $\mathbf{6}$ and $7.995 \mathrm{ppm}$ for $\mathbf{7}$ confirmed the presence of $\mathrm{N} \mathrm{NH} \mathrm{C}$ protons in the 6 and 7.

\section{In vitro evaluation of the antiproliferative activity}

\section{Structure activity relationship}

To evaluate the antiproliferative activity of these compounds, the $\mathrm{IC}_{50}$ values were determined in SMMC 7404, HeLa and MGC 7901 cancer cells by using a MTT assay according to the manufac turer's instructions. MTT is a compound that can be taken up by viable cells and reduced by a mitochondrial dehydrogenase form ing a formazan product in living cells. The absorbance of the forma zan product at $492 \mathrm{~nm}$ is in linear proportion to cell numbers. The results were summarized as $\mathrm{IC}_{50}$ values in $\mu \mathrm{mol} / \mathrm{L}$ in Table 1. 
Table 1

In vitro antiproliferative activities ( $\mathrm{IC}_{50}$ in $\mu \mathrm{mol} / \mathrm{L}$ ) of the compounds 2-9.

\begin{tabular}{llll}
\hline \multirow{2}{*}{ Compounds } & \multicolumn{2}{l}{ Carcinoma cell lines } & \\
\cline { 2 - 4 } & MGC 7901 & HeLa & SMMC 7404 \\
\hline $\mathbf{2}$ & 26.5 & 42.1 & 25.3 \\
$\mathbf{3}$ & 31.8 & 11.3 & 28.9 \\
$\mathbf{4}$ & 15.8 & 17.2 & 16.8 \\
$\mathbf{5}$ & 12.8 & 22.8 & 17.6 \\
$\mathbf{6}$ & 15.3 & 6.5 & 40.9 \\
$\mathbf{7}$ & 36.6 & 10.6 & 78.3 \\
$\mathbf{8}$ & $>100$ & 7.7 & 77 \\
$\mathbf{9}$ & 16.3 & 5.6 & 17.9 \\
Cisplatin & 6.7 & 10.1 & 23.2 \\
\hline
\end{tabular}

Apparently all steroidal lactams ( 2 9) displayed a distinct cyto toxicity against these cancer cells. Although the cytotoxic activity against MGC 7901 and SMMC 7404 cells was not significantly different between $4 N$ lactam 2 and $3 N$ lactam 3 or 5 and 9, 3 $N$ lactams showed a higher cytotoxicity against HeLa cells than $4 \mathrm{~N}$ lactams. Interestingly $4 \mathrm{~N}$ lactam 4 exhibited a high cytotox icity to all cancer cells tested, but the cytotoxic activity was remarkably deceased in $3 \mathrm{~N}$ lactam 8 although HeLa cells were sensitive to the compound.

Compounds 2 and 4 7, with same $4 N$ lactam structure and dif ferent types of 6 substituted groups, showed a distinct difference in their cytotoxicity against these cancer cells. The analogs $\mathbf{4}$ and $\mathbf{5}$, with a hydroxyl or a hydroximino at $C 6$, remarkably increased their cytotoxic activity against MGC 7901 and SMMC 7404 cells in comparison with the analogs 2 and 7, which have a carbonyl or semicarbazone groups at C 6 . Compounds $\mathbf{6}$ and $\mathbf{7}$ with a thio semicarbazone or semicarbazone groups at $C 6$ had a better cyto toxicity than compounds $\mathbf{2}, \mathbf{4}$ and $\mathbf{5}$ against HeLa cells. Here compounds 6, 8, 9 (6: $6.56 \mu \mathrm{mol} / \mathrm{L} ; 8: 7.76 \mu \mathrm{mol} / \mathrm{L} ; 9: 5.6 \mu \mathrm{mol} /$ L) were even more cytotoxic than cisplatin to HeLa cells (positive contrast: $10.1 \mu \mathrm{mol} / \mathrm{L}$ ).

\section{Conclusion}

We have prepared a series of 3 aza A homo 3 oxycholestane and 4 aza A homo 3 oxycholestane derivatives with different substituted groups at position 6 of the ring B. The antiproliferative activity of the synthesized compounds against SMMC 7404, HeLa and MGC 7901 cancer cells was investigated. All these compounds displayed a distinct cytotoxicity against these cancer cells. Our results revealed that the structures of functional groups at posi tion 6 on the steroidal ring are crucial for the $\mathrm{IC}_{50}$ value of antipro liferative activities of these compounds and the cytotoxic activity against MGC 7901 and SMMC 7404 cells was not significantly different between $4 \mathrm{~N}$ lactams and $3 \mathrm{~N}$ lactams when its 6 substi tuted group was a carbonyl or a hydroximino, but all $3 \mathrm{~N}$ lactams showed a higher cytotoxicity against HeLa cells than $4 \mathrm{~N}$ lactams. Our findings could provide new evidence showing the relationship between the chemical structure and biological activity and may be useful for the design of novel chemotherapeutic drugs.

\section{Acknowledgments}

The authors acknowledge the financial support of the Natural Science Foundation of Guangxi Province (Nos. 2010GXNSFD013 019 and 0832106).

\section{References}

[1] Festi D, Montagnani M, Azzaroli F, Lodato F, Mazzella G, Roda A, et al. Clinical efficacy and effectiveness of ursodeoxycholic acid in cholestatic liver diseases. Curr Clin Pharmacol 2007;2(2):155-77.

[2] Ifere GO, Barr E, Equan A, Gordon K, Singh UP, Chaudhary J, et al. Differential effects of cholesterol and phytosterols on cell proliferation, apoptosis and expression of a prostate specific gene in prostate cancer cell lines. Cancer Detect Prev 2009;32(4):319-28.

[3] Duha CY, Loa IW, Wang SK, Dai CF. New cytotoxic steroids from the soft coral Clavularia viridis. Steroids 2007;72:573-9.

[4] Malika IO, Maurice S. Recent advances in thiasteroids chemistry. Steroids 2006;71:1025-44.

[5] James RH. Steroids: partial synthesis in medicinal chemistry. Nat Prod Rep 2006;23:100-7.

[6] Chen SJ, Cui JG, Li Y, Fan LH. Recent advance of steroidal hydrazone with biological activities. Chin J Org Chem 2011;31(2):187-92.

[7] Zhang XJ, Cui JG, Li Y, Chen SJ. Recent advance in steroidal oximes with biological activity. Chin J Org Chem 2010;30(5):655-61.

[8] Javier P, Miriam R, Vanessa P, Beatriz A, Jaime R, Nélida S, et al. Synthesis and evaluation of new 6-hydroximinosteroid analogs as cytotoxic agents. Bioorg Med Chem 2007;15:4722-40.

[9] Mohamed EF, Gamal AE, Emad FE, Hanaa MR, Mohamed AT. Novel modified steroid derivatives of androstanolone as chemotherapeutic anti-cancer agents. Eur J Med Chem 2009;44:3936-46.

[10] Dominique B, Laïla EK, Monique C, Walae S, Mohamed K, Christos R, et al Synthesis of bile acid derivatives and in vitro cytotoxic activity with proapoptotic process on multiple myeloma (KMS-11), glioblastoma multiforme (GBM), and colonic carcinoma (HCT-116) human cell lines. Eur J Med Chem 2010;45:2912-8.

[11] Cui JG, Fan L, Huang LL, Liu HL, Zhou AM. Synthesis and evaluation of some steroidal oximes as cytotoxic agents: structure/activity studies (I). Steroids 2009;74(1):62-72.

[12] Cui JG, Fan L, Huang YM, Xin Y, Zhou AM. Synthesis and evaluation of some steroidal oximes as cytotoxic agents: structure/activity studies (II). Steroids 2009;74(12):989-95.

[13] Venetia K, Eleftheria M, Zafiroula I, Dionysios M, Manolis F, Anna K, et al. A new approach for evaluating in vivo anti-leukemic activity using the SCE assay: an application on three newly synthesised anti-tumour steroidal esters. Mutat Res 2003;535:79-86.

[14] Dimitrios TPT. Hybrid aza-steroid alkylators in the treatment of colon cancer Cancer Lett 2006;243:202-10.

[15] Natalija MK, Mira SB, Željko Ž, Mirjana DP, Zorica DJ, Vladimir DP. Synthesis of some steroidal oximes, lactams, thiolactams and their anti-tumor activities. Steroids 2007;72:406-14.

[16] Anna IK, Manolis AF, Evagelia SA, Athanasios P, George NP, Sotiris SN. Design, synthesis, and in vivo evaluation of the antileukemic activity of six new alkylating steroidal esters. Bioorg Med Chem 2008;16:5207-15.

[17] Dimitrios TPT, George DG, Catherine K, Athanasios P, Panayiotis K, Charalambos C. A D-homo-aza-androsterone alkylator in the treatment of breast cancer. Breast Cancer Res Treat 2006;97:17-31.

[18] Huang YM, Chen SJ, Cui JG, Gan CF, Liu ZP, Wei YL, et al. Synthesis and cytotoxicity of A-homo-lactam derivatives of cholic acid and 7-deoxycholic acid. Steroids 2011;76:690-4.

[19] Huang YM, Cui JG, Zhong ZG, Gan CF, Zhang WY, Song HC. Synthesis and cytotoxicity of 17a-Aza-D-homo-androster-17-one derivatives. Bioorg Med Chem Lett 2011;21:3641-3.

[20] Cui JG, Gan CF, Fan L. NMR spectral characteristic and conformation analysis of (3E)-cholest-4-en-3,6-dione-3-oxime and (6E)-cholest-4-en-3,6-dione-6oxime. J Instrum Anal 2008;27:523-6 [in Chinese]. 